\title{
The tip fracture of the Coopdech bronchial blocker during insertion in the patient with lung surgery
}

\author{
Deokkyu Kim, Ji-Seon Son, Hyungsun Lim, Jun Ho Lee, and Eun Joo Jang \\ Department of Anesthesiology and Pain Medicine, Chonbuk National University Medical School and Hospital, Jeonju, Korea
}

Bronchial blockers are simple alternative methods of facilitating placement of devices for one-lung ventilation. The Coopdech bronchial blocker (Daiken Medical, Osaka, Japan) has been clinically introduced to perform one-lung ventilation with a single-lumen tracheal tube [1]. The distal tip of the Coopdech bronchial blocker has a pre-formed angulation. We experienced a case of fracture of the tip of the Coopdech bronchial blocker during insertion for one-lung ventilation.

A 65-year-old female was scheduled for video-assisted thoracoscopic surgery and lung biopsy for suspected solitary pulmonary nodule in her right middle and lower lobes. Her medical history was unremarkable except for well-controlled asthma. The patient's airway examination revealed Mallampati II and a thyromental distance of 4-finger breadths. Her chest computerized tomography revealed no abnormality of the trachea or major bronchi. Her trachea was easily intubated with a $7.5 \mathrm{~mm}$ internal diameter (ID) single-lumen tracheal tube (HiLo oral/nasal tracheal tube, Mallinckrodt, Athlone, Ireland). A Coopdech bronchial blocker was then inserted into the tracheal tube, and the length of the blocker was about $40 \mathrm{~cm}$ from the tip of the blocker to the connector of the tracheal tube. A pediatric fiberoptic bronchoscope (FI-7RBS, Pentax Medical Company, Montvale, NJ, USA) was passed through the single-lumen tracheal tube, and the tip of the blocker was visualized between the carina and the tip of tracheal tube. Despite repeated attempts under bronchoscopic guidance, the blocker could only be inserted into the left main bronchus. After removing pediatric fiberoptic bronchoscope, the tip of the bronchial blocker was rotated to the right side and then advanced blindly into the right main bronchus. After advancing the blocker for $2-3 \mathrm{~cm}$, the resistance increased abruptly. Tip fracture of the bronchial blocker, which was folded backward, was visualized by reinserted pediatric fiberoptic bronchoscope. Subsequent attempts to withdraw the blocker failed because the bent tip hooked the tracheal tube. Under laryngoscopic guidance, subsequent attempts to remove both the tracheal tube and bronchial blocker also failed because the bent tip hooked the vocal cord. Both the tracheal tube and bronchial blocker were reinserted up to the carina, and the tip of the tracheal tube was positioned $2 \mathrm{~cm}$ above the carina, which was a relatively large space, with bronchoscopic guidance. The fractured tip of the bronchial blocker was rotated to the

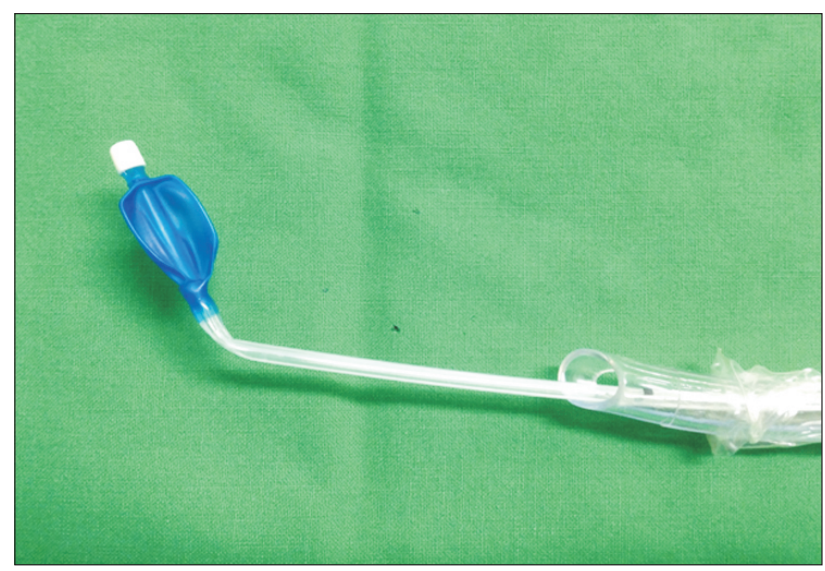

Fig. 1. The Coopdech bronchial blocker showing its fractured tip and the accompanying endotracheal tube.

Corresponding author: Ji-Seon Son, M.D., Ph.D., Department of Anesthesiology and Pain Medicine, Chonbuk National University Medical School and Hospital, 20, Geonji-ro, Deokjin-gu, Jeonju 561-712, Korea. Tel: 82-63-250-1246, Fax: 82-63-250-1240, E-mail: sjs6803@jbnu.ac.kr (c) This is an open-access article distributed under the terms of the Creative Commons Attribution Non-Commercial License (http:// creativecommons.org/licenses/by-nc/3.0/), which permits unrestricted non-commercial use, distribution, and reproduction in any medium, provided the original work is properly cited. 
right side, and then the bronchial blocker was pulled into the tracheal tube. The bronchial blocker was successfully removed without leaving behind any remnants. Consequently, the bronchial blocker that was removed revealed more marked angulation of the natural angle portion (Fig. 1). Then, a 35 Fr doublelumen tube was inserted instead of another Coopdech bronchial blocker for the fast progress of the operation. The surgery was performed successfully. The patient experienced no complications from this event.

In our patient, we performed repeated insertion into the left main bronchus under bronchoscopic guidance. Blind insertion may cause the tip fracture after withdrawing the pediatric fiberoptic bronchoscope. Venkataraju et al. [2] reported that partial fracture of the Coopdech bronchial tip occurred when attempting to place the bronchial blocker in the right main bronchus. They suspected that the angulated tip of the blocker may have been caught in the Murphy's eye of the tracheal tube. However, in our case, the cause of fracture was not accidental catching in the Murphy's eye of the tracheal tube.

In general, bronchial blockers are used with single-lumen tubes of $8.0 \mathrm{~mm}$ ID or more [3-5], but the Coopdech blocker was successfully used with a $7.5 \mathrm{~mm}$ ID single-lumen tube and a pediatric fiberoptic bronchoscope in an adult patient with tracheostomy stoma [5]. Moreover, the smallest recommended internal diameter of the endotracheal tube for use with the en- dotracheal blocker is $7.5 \mathrm{~mm}$ according to the manual of the Coopdech blocker. We wanted to use tubes with smallest diameter for preventing tube-induced complications. We suspect that poor control of the blocker caused repeated failure because the blocker was placed with a pediatric fiberoptic bronchoscope in a relatively small $7.5 \mathrm{~mm}$ ID single-lumen tube, and the inflated cuff of a single-lumen tube could be deviated into the trachea as the trachea and the lumen of the tube might not parallel. The blocker would have been placed in a better position if the bronchoscope was reinserted or the cuff of the single-lumen tube was deflated.

The Coopdech Bronchial Blocker, like the Univent tube, has a pre-formed angulation at its tip to facilitate insertion into the desired bronchus. Garcia-Guasch et al. [4] reported that the Coopdech bronchial blocker has more advantages such as a simple torque mechanism than Arndt bronchial blockers because it has a deflected tip. However, the Coopdech bronchial blocker is relatively more rigid for maintaining angulation, and it can be difficult to slide the blocker forward blindly because it is more likely to act as a wedge. The blocker should not be advanced if resistance is encountered.

We suggest that the Coopdech bronchial blocker should be inserted under continuous bronchoscopic guidance for the purpose of reducing the risk of tip fracture during its placement.

\section{References}

1. Ishizaki T, Yanagihara H, Isshiki A. One-lung ventilation using a new original connector: with a bronchial blocker and a single lumen tracheal tube. Masui 2003; 52: 191-4.

2. Venkataraju A, Rozario C, Saravanan P. Accidental fracture of the tip of the Coopdech bronchial blocker during insertion for one lung ventilation. Can J Anaesth 2010; 57: 350-4.

3. Han JI, KimDY, Lee GY, Kim CH. Comparison of the effects among three methods of bronchial blockade in one lung ventilation. Korean J Anesthesiol 2003; 44: 210-6.

4. Garcia-Guasch R, Flo A, de Castro PL. Coopdech bronchial blocker is useful in abnormalities of the tracheobronchial tree. J Cardiothorac Vasc Anesth 2010; 24: 735-6.

5. Uzuki M, Kanaya N, Mizuguchi A, Kurosawa S, Nakayama M, Omote K, et al. One-lung ventilation using a new bronchial blocker in a patient with tracheostomy stoma. Anesth Analg 2003; 96: 1538-9. 